\title{
LXXXIII. On some optical effects including refraction and rotation of the plane of polarization due to the scattering of light by electrons
}

\section{Sir J.J. Thomson O.M. P.R.S.}

To cite this article: Sir J.J. Thomson O.M. P.R.S. (1920) LXXXIII. On some optical effects including refraction and rotation of the plane of polarization due to the scattering of light by electrons, Philosophical Magazine Series 6, 40:240, 713-734, DOI: 10.1080/14786441208636182

To link to this article: http://dx.doi.org/10.1080/14786441208636182

冓 Published online: 08 Apr 2009.

Submit your article to this journal

Џll Article views: 8

Q View related articles $₫$

47 Citing articles: 3 View citing articles 진 


\section{$\left[\begin{array}{ll}713\end{array}\right]$}

LXXXIII. On some Optical Effects including Refraction and Rotation of the Plane of Polarization due to the Scattering of Light by Electrons. By Sir J. J. Тномsом, O.M., P.R.S. .*

$\mathrm{W}^{\mathrm{E}}$ begin by considering the radiation along the axis of $x$ emitted by the electrons in a very thin slab bounded by planes at right angles to this axis; all the electrons in the slab are supposed to be moving in the same way at the same instant.

If an electron $O$ has an acceleration $f$ in a direction at right angles to $\mathrm{OP}$ the electric force at $\mathrm{P}$ at the time $\mathrm{T}$ due to this acceleration is parallel to $f$ and equal to $-e f_{1} / c^{2}$. OP, where $f_{1}^{\prime}$ is the acceleration at the time $\mathrm{T}-\mathrm{OP} / c, e$ being the charge on the electron and $c$ the velocity of light. Let $\mathrm{P}$ be a point in front of the slab on the axis of $x$; if the acceleration parallel to $y$ of an electron $Q$ in the slab is $d^{2} y / d t^{2}$, the electron will produce an electric force parallel to $y$ at $\mathrm{P}$ at the time ' $\mathrm{T}$ equal to

$$
-\frac{e}{c^{2} \cdot \mathrm{PQ}}\left(\frac{d^{2} y}{d t^{2}}\right)_{\mathrm{T}-\frac{\mathrm{PQ}}{c}},
$$

provided $P Q$ is at right angles to $y$. Since the optical effects at $\mathrm{P}$ of a plane wave arise from the parts adjacent to $O$, where $O$ is the foot of the perpendicular let fall from $\mathrm{P}$ on the plane of the wave, we may make this supposition without loss of generality.

If $\mathrm{D}$ is the thickness of the slab, $\sigma$ the density of the electrons, $\mathrm{Y}_{1}$ the electric force parallel to $y$ at $\mathrm{P}$ at the time $\mathrm{T}$ is given by the equation

$$
\mathrm{Y}_{1}=-\frac{e \cdot \sigma \mathrm{D}}{c^{2}} \int \frac{1}{r}\left(\frac{d^{2} y}{d t^{2}}\right)_{\mathrm{T}-\frac{r}{c}} 2 \pi \rho d \rho,
$$

where

$$
\rho=\mathrm{OQ}, \quad r=\mathrm{PQ} \text {. }
$$

Now

$$
r^{2}=\rho^{2}+\mathrm{OP}^{2}
$$

hence

$$
r d r=\rho d \rho,
$$

and therefore

$$
\mathrm{Y}_{1}=-\frac{2 \pi e \sigma \mathrm{D}}{c^{2}} \int\left(\frac{d^{2} y}{d t^{2}}\right)_{\mathrm{I}-\frac{p}{\hat{c}}} d v .
$$

* Communicated by the Author.

Phil. Llag. S. 6. Vol. 40. No. 240. Dec. 1920. 
Let

$$
\omega=t-\frac{r}{c},
$$

as throughout the integration $t$ is constant

$$
d r=-c \cdot d \omega \text {. }
$$

Since at the same instant the accelerations of all the electrons in the slab are equal,

hence

$$
\begin{gathered}
y=f(t), \\
\left(\frac{d^{2} y}{d t^{2}}\right)_{t-\frac{r}{c}}=\frac{d^{2} \cdot f(\omega),}{d \omega^{2}}, \\
\mathrm{Y}_{1}=\frac{2 \pi}{c} \epsilon \sigma \mathrm{D} \int \frac{d^{2} f^{\prime}(\omega)}{d \omega^{2}} d \omega \\
=\frac{2 \pi}{c} e \sigma \mathrm{D}\left[\frac{d f}{d \omega}\right]_{\omega_{1}}^{\omega_{2}} .
\end{gathered}
$$

The limits of $\omega$ are $t-\frac{x}{c}$ where $c=0 \mathrm{P}$, and $t-\frac{\mathrm{R}}{c}$ where $R$ is the greatest value of $P Q$; when $R$ is so large that $t-\mathrm{R} / c$ corresponds to a time before the electrons began to be affected by the light wave,

so that

$$
\begin{gathered}
\left(\frac{d f}{d \omega}\right)_{\omega_{2}}=0, \\
\mathrm{Y}_{1}=-\frac{2 \pi}{c} e \sigma \mathrm{D}\left(\frac{d f}{d \omega}\right)_{t-\frac{x}{c}} \\
=-\frac{2 \pi}{c} e \sigma \mathrm{D} \cdot v_{t-\frac{x}{c}},
\end{gathered}
$$

where $v_{t-\frac{x}{c}}$ is the velocity of an electron parallel to $y$ at the time $t-x / c$. If $k=\frac{2 \pi}{c} e \sigma \mathrm{D}$, we have

$$
\mathrm{Y}_{1}=-k v_{t-\frac{x}{c}} \cdot \cdot \cdot \cdot \cdot \cdot \cdot
$$

Similarly $Z_{1}$ the force parallel to $z$ will be given by

$$
\mathrm{Z}_{1}=-k w_{t-\frac{x}{c}}
$$

where $w_{t=\frac{x}{c}}$ is the velocity of an electron parallel to $z$ at the time $t-\frac{x}{c}$. 
Let us now consider a plane wave travelling in the positive direction of the axis of $x$, let $f(x, t)$ be the value of the electric force parallel to $Z$ at the place $x$ and the time $t$. Consider now how the force at the point $x+\delta x$, at the time $t+\delta x / c$, differs from that at $x_{1}$ and $t_{1}$. The force which is at $x$ at the time $t$ will be found at $x+d x$, at the time $t+\delta x / c$, and in addition to this force there will be found at $x+\delta x$ the force due to the secondary waves which come from the electrons in the slab $\delta x$. This force, as we have just seen, is equal to

$$
-\frac{2 \pi}{c} e \sigma\left(\frac{d z}{d t}\right) \delta x
$$

Hence we have

$$
f(x+\delta x, t+\delta \cdot c / c)=f(x, t)-\frac{2 \pi}{a} e \sigma\left(\frac{d z}{d t}\right) \delta x .
$$

This equation is equivalent to

$$
\frac{d f}{d x}+\frac{1}{c} \cdot \frac{d \dot{f}}{d t}=-\frac{2 \pi}{c} e \sigma\left(\frac{d z}{d t}\right)
$$

If the equation of motion of an electron in the slab is

$$
m\left(\frac{d^{2} z}{d t^{2}}+n^{2} z\right)=e f
$$

and if $f$ varies as $\epsilon^{\imath p t}$, then

$$
z=\frac{e f}{m\left(n^{2}-p^{2}\right)},
$$

and equation (2) becomes

$$
\begin{aligned}
& \frac{d f}{d v}+\frac{1 d f^{\prime}}{c t t}=-\frac{2 \pi}{c}-\frac{e^{2} \sigma}{m\left(n^{2}-p^{2}\right)} d t \\
& \text { or } \quad \frac{d \dot{f}}{d x}+\frac{1}{c^{\prime}} \frac{d j}{d t}=0, \quad . \quad . \quad . \quad . \\
& \text { if } \quad \frac{1}{c^{\prime}}=\frac{1}{c}+\frac{2 \pi}{c} \cdot \frac{e^{2}}{m} \frac{\sigma}{n^{2}-p^{2}} . \quad . \quad . \quad . \quad \text {. }
\end{aligned}
$$

The solution of (2) is

$$
f=\phi\left(x-i^{\prime} t\right)
$$

representing a disturbance propagated with a velocity $c^{\prime}$. If $\mu$ is the refractive index,

hence by (4)

$$
\mu=c / c^{\prime},
$$

$$
\mu=1+\frac{2 \pi e^{2}}{m} \cdot \frac{\sigma}{\left(n^{2}-p^{2}\right)} .
$$


This is the well-known expression given by Lorentz, If the electrons have not.all the same frequency, then if $\sigma_{s}$ is the density of those with the frequency $n_{s}$, we can show without difficulty that

$$
\mu=1+2 \pi \frac{e^{2}}{m} \Sigma \frac{\sigma_{s}}{m_{s}^{2}-p^{2}}
$$

This expression has been used by Drude and otbers to determine the number of electrons in the atoms. In using it for this purpose, however, it is necessary to take account of some considerations which may be illustrated by the following example. Let us consider the case of an electron in an atom of such a character that the displacement of the electron by an electric force is not in general in the direction of the electric force. There will be three directions fixed in the atom such that a force along one of these directions produces a displacement in the same direction. If these directions are taken as the axes of $\xi, \eta, \zeta$, the equations of motion of the electron may be written

$$
\begin{aligned}
& m\left(\frac{d^{2} \xi}{d t^{2}}+n_{1}^{2} \xi\right)=e \mathrm{~F}_{\xi}, \\
& m\left(\frac{d^{2} \eta}{d t^{2}}+n_{2}^{2} \eta\right)=e \mathrm{~F}_{\eta}, \\
& m\left(\frac{d^{2} \zeta}{d t^{2}}+n_{3}^{2} \zeta\right)=e \mathrm{~F}_{\zeta},
\end{aligned}
$$

where $\mathrm{F}_{\xi}, \mathrm{F}_{\eta}, \mathrm{F}_{\zeta}$ are the components of the electric force in the directions $\xi, \eta, \zeta$ respectively. Let the axes of $\xi, \eta, \zeta$ cut a sphere at the points $A, B, C$, and the axis of $z$ at the point $Z$; then, if $\angle Z C=\theta$, and $\phi$ is the angle between $Z C$ and $\mathrm{CA}$, an electric force $\mathrm{Z}$ in the light wave will be equivalent to the forces

$$
\mathrm{F}_{\xi}=\mathrm{Z} \cos \theta, \quad \mathrm{F}_{\eta}=\mathrm{Z} \sin \theta \sin \phi, \quad \mathrm{F}_{\zeta}=-\mathrm{Z} \sin \theta \cos \phi,
$$

and if $Z$ varies as $\epsilon^{\imath p t}$, we get from the equations of motion of the electron

$$
\begin{gathered}
\xi=-\frac{e}{m} \frac{\mathrm{Z} \sin \theta \cos \phi}{n_{1}^{2}-p^{2}}, \quad \eta=\frac{e}{m} \frac{Z \sin \frac{\theta \sin \phi}{n_{2}{ }^{2}-p^{2}},}{\zeta=\frac{e}{m} \frac{Z \cos \theta}{n_{3}^{2}-p^{2}} .}
\end{gathered}
$$

The displacement resolved along the axis of $z$ is thus

$$
\frac{e}{m} \mathrm{Z}\left(\frac{\cos ^{2} \theta}{n_{3}^{2}-p^{2}}+\frac{\sin ^{2} \theta \sin ^{2} \phi}{n_{2}^{2}-p^{2}}+\frac{\sin ^{2} \theta \cos ^{2} \phi}{n_{1}^{2}-p^{2}}\right) \text {. }
$$


If the atoms are orientated uniformly, the mean value of this expression is

$$
\text { Z } \frac{e}{m}\left(\frac{1}{n_{1}^{2}-p^{2}}+\frac{1}{n_{2}^{2}-p^{2}}+\frac{1}{n_{3}^{2}-p^{2}}\right) \text {. }
$$

So that if $\sigma$ is the density of the electrons, the value of $\mu$ the refractive index will be

$$
1+\frac{2 \pi}{3} \frac{e^{i} \sigma}{m}\left(\frac{1}{n_{1}^{2}-p^{2}}+\frac{1}{n_{2}^{2}-p^{2}}+\frac{1}{n_{3}^{2}-p^{2}}\right) .
$$

Thus if $n_{2}$ and $n_{3}$ were very large compared with $n_{1}$,

$$
\mu=1+\frac{2 \pi}{3} \frac{e^{2} \sigma}{m} \frac{1}{n_{1}^{2}-p^{2}}
$$

thus the true value of $\sigma$ would be three times that obtained by the usual formula

$$
\mu=1+2 \pi \frac{e^{2}}{m} \frac{\sigma}{n^{2}-p^{2}} .
$$

The usual formula is only true when the displacement of the electron is always in the direction of the resultatit force; when this is so $n_{1}=n_{2}=n_{3}$, and the expression for $\mu$ takes the usual form.

\section{Conduction in Metals.}

If we suppose that the velocity of the electrons is proportional to the electric force, then

$$
\frac{d z}{d t}=\frac{(f f}{e \sigma}
$$

where $C$ is the specific conductivity of the metal; for $e \sigma \frac{d z}{d t}$ is the current through unit area. Using this value of $d z / d t$ equation (2) gives

$$
\frac{d f}{d x}+\frac{1}{c} \frac{d f}{d t}=-\frac{2 \pi \mathrm{C}}{c} f
$$

The solution of which is

$$
j=\epsilon^{-2 \pi \mathrm{C} x} " \phi(x-c t) .
$$

Since the intensity of the radiation is proportional to $f^{\prime 2}$, the rate of decay of this intensity is

$$
e^{-\frac{4 \pi \mathrm{C}}{6} \cdot x}
$$

so that the coefficient of absorption is equal to $4 \pi(\%)$ 


\section{Changes produced by the Electrons on the type of Polarization.}

We see from equation (1) that if the electrons acquire from the primary wave a finite average velocity in any direction, secondary wares with the electric force in that direction will be emitted. Thus unless the displacement of the electrons due to the electric force in the primary wave is in the direction of that force, the electric force in the secondary waves will not be in the same direction as that in the primary, and the mixture of primary and secondary waves will differ in the state of the polarization from the primary light. The change may be one or other of two types: (1) the mixed light may differ from the primary by being elliptically polarized; or (2) it may still be plane polarized, but the plane of polarization may be rotated.

$Y$ the force in the scattered light is by equation (1) proportional to $d y / d t$ : hence, if $y$ is in the same phase as $Z$, the force in the primary light, $d y / d t$ and therefore $\mathrm{Y}$ will differ in phase from $Z$ by a quarter of a period, so that the mixture of primary and scattered light will be elliptically polarized. If, however, $d y / d t$ is in the same phase as $Z$, $Y$ and $Z$ will be in the same phase and the mixture of scattered and primary light will be plane polarized, though the plane of polarization will not coincide with that of the primary light.

Unless the electrons in an atom are distributed in an exceptionally symmetrical way, a force parallel to $z$ will produce a displacement parallel to $y$, but in cases similar to that discussed on page 716 the $y$ displacement will be in the same phase as $Z$, and $d y / d t$ will differ in phase from $Z$ by a quarter of a period and the light will be elliptically polarized. The amount of ellipticity in the polarization will depend upon the orientation of the atoms or molecules, and in non-crystalline substances there may be as many molecules producing a positive effect as there are producing a negative one, so that the aggregate effect may be nil and the light will continue to be plane polarized. Thus in the case considered on page 716 the displacement pamllel to $y$ produced by the force $Z$ is

$$
\begin{aligned}
\xi(\cos \theta & \sin \phi \cos \psi+\cos \phi \sin \psi) \\
& +\eta(-\cos \theta \sin \phi \sin \psi+\cos \phi \cos \psi)+\zeta(\sin \theta \sin \phi),
\end{aligned}
$$

where $\theta$ and $\phi$ have the same values as before, and $\psi$ is the angle between $\mathrm{ZC}$ and $\mathrm{ZX}$. Substituting the values of 
$\xi, \eta, \zeta$ given on page 716 , we find that the displacement parallel to $y$ is

$$
\begin{aligned}
& \frac{\mathrm{Z} e}{m}\left\{\frac{1}{n_{1}^{2}-p^{2}} \sin \theta \sin \psi(\cos \theta \sin \phi \cos \psi+\cos \phi \sin \psi)\right. \\
& -\frac{1}{n_{2}^{2}-p^{2}} \sin \theta \cos \psi(-\cos \theta \sin \phi \sin \psi+\cos \phi \cos \psi) \\
& \left.\quad+\frac{1}{n_{3}^{2}-p^{2}} \cdot \cos \theta \sin \theta \sin \phi\right\} .
\end{aligned}
$$

If there is no order in the arrangement of the atoms or molecules, we see that the average value of each term is zero, so that the light would not be elliptically polarized.

\section{Magnetic Rotation of the Plane of Polarization.}

An important case when an electric force acting on an electron may produce motion at right angles to itself is when the electrons are exposed to a magnetic field parallel to the direction of propagation of the light. Let $\mathrm{H}$ be the magnitude of the magnetic force, $y$ and $z$ the displacements of the electron parallel to $y$. and $z$ respectively, $Z$ the electric force in the light wave. The equations of motion of the electron will be of the form

$$
\begin{aligned}
& m\left(\frac{d^{2} z}{d t^{2}}+n^{2} z\right)=\mathrm{Z} e+\mathrm{H} e \frac{d y}{d t} \\
& m\left(\frac{d^{2} y}{d t^{2}}+n^{2} y\right)=-\mathrm{H} e \frac{d z}{d t} .
\end{aligned}
$$

Hence, neglecting terms in $\mathrm{H}^{2}$, we find if $Z$ varies as $\epsilon^{t p^{t}}$,

$$
\frac{d y}{d t}=\frac{\mathrm{ZH} e^{2} p^{2}}{m^{2}\left(\bar{n}^{2}-p^{2}\right)^{2}}
$$

We see from equation (1) that this involves the production of a wave in which the electric torce is parallel to $y$, and that $\mathrm{Y}$ the magnitude of this force for a slab of thickness $\mathrm{D}$ is given by the equation

$$
\begin{aligned}
\mathrm{Y} & =-2 \pi \frac{\sigma e}{c} \mathrm{D} \frac{d y}{d t} \\
& =-\frac{2 \pi \sigma e^{3}}{m^{2} c} \cdot \mathrm{D} \cdot \frac{p^{2}}{\left(n^{2}-p^{2}\right)^{2}} \cdot \mathrm{H} \cdot \mathrm{Z} .
\end{aligned}
$$

In traversing the distance $D$ the plane of polarization is twisted through the angle $\mathrm{Y} / \mathrm{Z}$, or

$$
\frac{2 \pi \sigma \cdot e^{3}}{m^{2} c} \frac{p^{2}}{\left(n^{2}-p^{2}\right)^{2}} \text { H. D. }
$$


If $\mu$ is the refractive index,

$$
\mu=1+\frac{2 \pi e^{2} \sigma}{m\left(n^{2}-p^{2}\right)} ;
$$

hence the angle through which the plane of polarization is twisted is equal to

this can be written as

$$
\mu-1 \cdot \frac{e}{m} \frac{p^{2}}{n^{2}-p^{2}} \text { H.D; . . . . . }
$$

$$
\frac{p}{2 c} \frac{e}{m} \frac{d \mu}{d p} \mathrm{H} . \mathrm{D}, . . \quad . \quad . \quad . \quad .
$$

which agrees with the expression given by Becquerel.

$\mathrm{He} / m$ is the angular velocity with which an electron would describe a spiral round the line of magnetic force $H$; let this be denoted by $\omega$, then if $\tau$ is the time the light takes to pass across the slab $D$, the angle through which the plane of polarization is rotated in this time is

$$
(\mu-1) \frac{p^{2}}{n^{2}-p^{2}} \omega \tau
$$

Thus when the atoms have only one intrinsic frequency the rotation is given by a very simple expression. If $n$ is large compared with $p$, the rotation is proportional to $(\mu-1) p^{2} / n^{2}$, and is thus proportional to the square of the frequency: a result which is a rough approximation to the truth in a considerable number of cases.

If, as indicated by equation (7), the rotation can be expressed as a function of the dispersion, it follows that no information as to the structure of molecules can be obtained by experiments on magnetic rotation which cannot be obtained by experiments on dispersion.

\section{Rotation of the Plane of Polarisation by substances such as quartz or sugar solution.}

We cannot explain this rotation if we consider isolated electrons in an atom, but we shall see that we could account for it by a system of electrons held so firmly in position that they act somewhat as a rigid body, a force acting on one electron displacing the whole system of electrons.

We shall suppose as before that the primary beam is travelling along the axis of $x$, that it is plane polarized, and that the electrical force in it is parallel to $z$. Let the coordinates of an electrical charge, electron or positive particle, be $x_{s}, y_{s}, z_{s}$. If $Z_{s}$ is the electrical force in the primary wave at this point, the moment about the axis of $x$ of the forces acting on the electrical charges in the atom is $\sum e Z_{s} y_{s}$. 
If the dimensions of the molecule are small compared with the wave-length of the light, we have, very approximately,

$$
\mathrm{Z}_{s}=\mathrm{Z}_{0}+\frac{d \mathrm{Z}_{0}}{d_{i}} x_{s}
$$

where $Z_{0}$ is the value of $Z$ at the origin $O$ a point inside the molecule. Thus the moment of the forces about the axis of $x$ is

$$
Z_{0} \Sigma e y_{s}+\frac{d Z_{0}}{d x} \Sigma e x_{s, y_{s}}
$$

Similarly there is a couple around the axis of $y$ equal to

$$
-\mathrm{Z}_{0} \Sigma e x_{s}-\frac{d \mathrm{Z}_{0}}{d x} \Sigma e x_{s}^{2} \text {. }
$$

These couples acting on the system of electrical charges considered as a rigid body will cause it to rotate, and thus move the individual charges. If the system is not symmetrical the average velocity of the charges parallel to $y$ may be finite, and hence, by equation (1), give rise to an electric wave in which the electrical force has a component parallel to $\mathrm{Y}$. 'The phase of this force is the same as that of $\Sigma e \frac{d y}{d t}$. The amount of rotation of the plane of rotation will depend entirely on that part of the force $\mathrm{Y}$ which is in the same phase as $Z$. There may be other parts differing in phase from $Z$ by a quarter of a period; these will affect the ellipticity of the polarization, but not the rotation.

Though the values of $d y / d t$ for the different electrical charges may be all in the same phase, yet, since the charges are not all in the wave-front, the secondary waves from them will not, when they arrive at a point, be all in the same phase. Thus, if $x_{s}$ be the $x$ coordinate of one of the particles, the phase of a vibration due to this particle relative to one starting from the origin will be accelerated by $x_{s} \frac{2 \pi}{\lambda}$, so that, if the velocity of this particle were represented by

$$
\cos \frac{2 \pi}{\lambda}\left(v t-x_{0}\right)
$$

the electric force due to it would have the phase corresponding to

$$
\cos _{\lambda}^{2 \pi}\left(r t-x_{0}+v_{s}\right)
$$

and would be represented, since $x_{s} / \lambda$ is small, by

$$
\cos \frac{2 \pi}{\lambda}\left(v t-x_{0}\right)-x_{s} \frac{2 \pi}{\lambda} \sin \frac{2 \pi}{\lambda}\left(r t-x_{0}\right) .
$$


Similarly, a term in $\frac{d y}{d t}$ represented by $\sin \frac{2 \pi}{\lambda}\left(v t-x_{0}\right)$ would give rise to vibrations represented by

$$
\sin \frac{2 \pi}{\lambda}\left(v t-x_{0}\right)+x_{s} \frac{2 \pi}{\lambda} \cos \frac{2 \pi}{\lambda}\left(v t-x_{0}\right) .
$$

Treating the collection of electrical charges as a rigid body, we proceed to calculate the value of $\sum e \frac{d y}{d t}$ due to the rotation of the molecule produced by the couples arising from the electrical forces in the light wave.

Let the principal axes of inertia of the molecule be taken as the axes of $x^{\prime}, y^{\prime}, z^{\prime}$; let $\left(l_{1}, m_{1}, n_{1}\right),\left(l_{2}, m_{2}, m_{2}\right),\left(l_{3}, m_{3}, n_{3}\right)$ bo the direction cosines of those axes with respect to the fixed axes $x, y, z$.

Then the moment of the forces about the axis of,$r^{\prime}$ is

$$
\Sigma_{e}\left(Z_{0}+\frac{d Z}{d x} \cdot x\right)\left(n_{3} y^{\prime}-n_{2} z^{\prime}\right)
$$

Now

$$
x=l_{1}{ }^{\eta^{\prime}}+l_{2} y^{\prime}+l_{3} z^{\prime},
$$

hence the moment of the forces about $x^{\prime}$

$$
\begin{gathered}
=Z_{0}\left(n_{3} \Sigma e y^{\prime}-n_{2} \Sigma e z^{\prime}\right)+\frac{d Z_{0}}{d x}\left(n_{3} \mathrm{M}-n_{2} \mathrm{~N}\right) . \\
\mathrm{L}=\Sigma_{2} e\left(l_{1} x^{\prime} x^{\prime}+l_{2} x^{\prime} y^{\prime}+l_{3} x^{\prime} z^{\prime}\right), \\
\mathrm{M}=\Sigma_{e}\left(l_{1} x^{\prime} y^{\prime}+l_{2} y^{\prime} y^{\prime}+l_{3} y^{\prime} z^{\prime}\right), \\
\mathrm{N}=\Sigma_{e} e\left(l_{1} x^{\prime} z^{\prime}+l_{2} y^{\prime} z^{\prime}+l_{3} z^{\prime} z^{\prime}\right) .
\end{gathered}
$$

Similarly the moment of the forces about the axes of $y^{\prime}$ and $z^{\prime}$ are respectively

$$
\begin{gathered}
\mathrm{Z}_{0}\left(n_{1} \Sigma e z^{\prime}-n_{3} \Sigma e x^{\prime}\right)+\frac{d Z_{0}}{d x^{\prime}}\left(n_{1} \mathrm{~N}-n_{3} \mathrm{~L}\right) \\
\mathrm{Z}_{0}\left(n_{2} \Sigma e x^{\prime}-n_{1} \Sigma e y^{\prime}\right)+\frac{d Z_{0}}{d t^{2}}\left(n_{2} \mathrm{~L}-n_{1} \mathrm{M}\right) . \\
\mathrm{Z}_{0}=\cos \frac{2 \pi}{\lambda}\left(a t-x_{0}\right) .
\end{gathered}
$$

Let

If $\mathrm{A}, \mathrm{B}, \mathrm{C}$ are the moments of inertia of the molecule about the axes $x^{\prime}, y^{\prime}, z^{\prime}$, and $\omega_{1}, \omega_{2}, \omega_{3}$ the angular velocities about these axes, we have, if we retain only the first powers of $\omega_{1}, \omega_{2}, \omega_{3}$, and suppose that the only forces acting on the 
molecule are those due to the forces in the electrical wave,

$$
\begin{aligned}
\mathrm{A} \frac{d \omega_{1}}{d t}=\cos \frac{2 \pi}{\lambda}(c t & \left.-x_{0}\right)\left(n_{3} \Sigma e y^{\prime}-n_{2} \Sigma e z^{\prime}\right) \\
& +\frac{2 \pi}{\lambda} \cdot \sin \frac{2 \pi}{\lambda}\left(c t-\omega_{0}\right)\left(n_{3} \mathrm{M}-n_{2} \mathrm{~N}\right),
\end{aligned}
$$

so that

$$
\begin{aligned}
A \omega_{1}=-\frac{\lambda}{2 \pi} t^{\prime} & \sin \frac{2 \pi}{\lambda}\left(c t-i_{0}\right)\left(n_{3} \Sigma e y^{\prime}-n_{2} \Sigma e z^{\prime}\right) \\
& -\frac{1}{c} \cos \frac{2 \pi}{\lambda}\left(t t-i_{0}\right)\left(n_{3} \mathbf{M}-n_{2} \mathrm{~N}\right),
\end{aligned}
$$

with symmetrical expressions for $\mathrm{B} \omega_{2}, \mathrm{C} \omega_{3}$.

The velocity of a particle due to these rotations has for components parallel to $x^{\prime}, y^{\prime}, z^{\prime}$,

$$
\omega_{2} z^{\prime}-\omega_{3} y^{\prime}, \quad \omega_{3} x^{\prime}-\omega_{1} z^{\prime}, \quad \omega_{1} y^{\prime}-\omega_{2} x^{\prime}:
$$

hence the velocity parallel to the fixed axis $y$ is

$$
m_{1}\left(\omega_{2} z^{\prime}-\omega_{3} y^{\prime}\right)+m_{2}\left(\omega_{3} r^{\prime}-\omega_{1} z^{\prime}\right)+m_{3}\left(\omega_{1} y^{\prime}-\omega_{2} x^{\prime}\right) .
$$

$\mathrm{Y}$ the electric force parallel to $y$ is, as we see by equation (1), proportional to the sum of these expressions.

Now to find the rotation we require the term in $\mathrm{Y}$ which is of the same phase as $Z, i$. $e$. we require the term in $\cos \frac{2 \pi}{\lambda}(c t-a)$ and do not need that in $\sin \frac{2 \pi}{\lambda}(c t-x)$.

But, as we have explained, owing to the different positions of the moving electrical charge, a term $\cos \frac{2 \pi}{\lambda}\left(c t-x_{0}\right)$ in the expression for the velocity of a particle whose coordinate is $x_{r}$ will give rise to a term

$$
\cos \frac{2 \pi}{\lambda}\left(c t-x_{0}\right)-x_{r} \cdot \frac{2 \pi}{\lambda} \sin \frac{2 \pi}{\lambda}\left(c t-x_{v}\right)
$$

in the expression for the electric force; while $\sin \frac{2 \pi}{\lambda}\left(c t-x_{0}\right)$ will give rise to

$$
\sin \frac{2 \pi}{\lambda}\left(c t-x_{0}\right)+x_{r} \frac{2 \pi}{\lambda} \cos \frac{2 \pi}{\lambda}\left(c t-x_{0}\right) .
$$

Remembering this we see that the contribution of the $r$ th 
charge to the term in the expression for $\mathrm{Y}$ proportional to $\cos \frac{2 \pi}{\lambda}\left(c t-x_{0}\right)$ is proportional to

$$
\begin{aligned}
& -\frac{\left(m_{3} u_{r}^{\prime}-m_{2} z_{r}^{\prime}\right)}{\mathrm{A} c}\left\{\left(n_{3} \mathrm{M}-n_{2} \mathrm{~N} ;-x_{r}\left(n_{3} \Sigma y^{\prime}-n_{2} \Sigma z^{\prime}\right)\right\}\right. \\
& -\frac{\left(m_{1} z_{r}^{\prime}-m_{3} x_{r}^{\prime}\right)}{\mathrm{B} r}\left\{\left(n_{1} \mathrm{~N}-n_{3} \mathrm{~T}\right)-x_{r}\left(n_{1} \Sigma z^{\prime}-n_{3} \Sigma x^{\prime}\right)\right\} \\
& -\frac{\left(m_{2} a_{r}^{\prime}-m_{1} y_{r}^{\prime}\right)}{(\mathrm{C} e}\left\{\left(n_{2} \mathrm{~L}-n_{1} \mathrm{M}\right)-x_{r}\left(n_{2} \Sigma x^{\prime}-n_{1} \Sigma y^{\prime}\right)\right\} .
\end{aligned}
$$

Thus $Q$ the contribution of the whole molecule will bo given by the equation

$$
\begin{aligned}
& \mathrm{Q}=-\frac{1}{\mathrm{~A} c}\left\{\left(m_{3} \Sigma e y^{\prime}-m_{2} \Sigma e z^{\prime}\right)\left(n_{3} \mathrm{M}-n_{2} \mathrm{~N}\right)\right. \\
& \left.-\left(n_{3} \Sigma e y^{\prime}-n_{2} \Sigma e z^{\prime}\right)\left(m_{3} \mathrm{M}-n_{2} \mathrm{~N}\right)\right\} \\
& -\frac{1}{B i}\{\ldots \ldots\} \\
& -\frac{1}{\mathrm{C}}\{\ldots, \ldots,\} \\
& =-\frac{1}{\mathrm{~A}} e^{\left\{m_{2} n_{3}-m_{3} n_{2}\right\}\left(\mathrm{N} \Sigma e y^{\prime}-\mathrm{M} \Sigma e^{\prime}\right)} \\
& -l_{1}\{\ldots \ldots\}-\frac{1}{B_{c}}\{\ldots \ldots\} \\
& =-\frac{l_{1}}{A}\left(\mathrm{~N} \Sigma e y^{\prime}-M \Sigma e z^{\prime}\right)-\frac{l_{2}}{B c}\left(\mathrm{~L} \Sigma e z^{\prime}-\mathrm{N} \Sigma e x^{\prime}\right) \\
& -\frac{l_{3}}{\mathrm{C} t}\left\{\mathrm{M} \Sigma e x^{\prime}-\mathrm{L} \Sigma e y^{\prime}\right\} \text {. }
\end{aligned}
$$

If there are $\sigma$ of these molecules per unit volume, the rotation of the plane of polarization per unit path is by equation (1) equal to

$$
2 \pi Q{ }^{\sigma} \cdot . . . \quad . \quad .
$$

We must distinguish between the cases (1) when the molecules are in the gaseous state or in solution, and (2) when they form parts of a crystal. In case (2) we may suppose that all the molecules are orientated in the same way so that $l_{1}, l_{2}, l_{3}$ are the same for all molecules; then equation (8) will give the rotation for light moving in the direction determined by $l_{1}, l_{2}, l_{3}$, which are the direction cosines of the path of the light relative to the axes of the 
crystal. In case (1) the values of $l_{1}, l_{2}, l_{3}$ will vary from molecule to molecule. If the orientation of the molecules is quite irregular, the mean values of $l_{1}{ }^{2}, l_{2}{ }^{2}, l_{3}{ }^{2}$ are each $1 / 3$, while those of $l_{1} l_{2}, l_{1} l_{3}, l_{2} l_{3}$ are zero: hence $\bar{Q}$ the mean value of $Q$ is given by

$$
\begin{aligned}
\overrightarrow{\mathrm{Q}}= & -\frac{1}{3 \mathrm{~A} c}\left\{\Sigma e x^{\prime} z^{\prime} \Sigma e y^{\prime}-\Sigma_{e} x^{\prime} y^{\prime} \cdot \Sigma e z^{\prime}\right\} \\
& -\frac{1}{3 \mathrm{~B} c}\left\{\Sigma e x^{\prime} y^{\prime} \Sigma e z^{\prime}-\Sigma e y^{\prime} z^{\prime} \cdot \Sigma e x^{\prime}\right\} \\
& -\frac{1}{3 C}\left\{\sum_{e} y^{\prime} z^{\prime} \Sigma e x^{\prime}-\Sigma e x^{\prime} z^{\prime} . \Sigma e y^{\prime}\right\},
\end{aligned}
$$

and the rotation for the solution is

$$
2 \pi \bar{Q}_{c}^{\sigma} \text {. . . . . . . . }
$$

I obtained a similar expression, by a different method, for this rotation in a paper published in the Proceedings of the Cambridge Philosophical Society, xiv. p. 313 (1907).

We see from the expression for $\bar{Q}$ that if it is to be finite the molecule must be very unsymmetrical.

For $\bar{Q}$ vanishes

(1) if the molecule hats dynamical symmetry, for then $\mathrm{A}=\mathrm{B}=\mathrm{C}$;

(2) if the centre of the olectrical charges coincides with the centre of mass, for then

$$
\Sigma_{e} x^{\prime}=\Sigma_{e y^{\prime}}=\Sigma_{e z^{\prime}}=0 ;
$$

(3) if the principal axes of inertia coincide with axes of symmetry of the electrical charges, for then

$$
\Sigma e x^{\prime} y^{\prime}=\Sigma e x^{\prime} z^{\prime}=\Sigma e y^{\prime} z^{\prime}=0 ;
$$

(4) if $\Sigma e y^{\prime} \Sigma e x^{\prime} z^{\prime}=\Sigma_{e} e z^{\prime} \Sigma e x^{\prime} y^{\prime}=\Sigma e x^{\prime} \Sigma e y^{\prime} z^{\prime}$.

This relation would be fulfilled if the electrical charges formed a geometrically symmetrical system, even though the masses might not be symmetrical. For such a system

$$
\Sigma e x^{\prime} z^{\prime}=\Sigma e x^{\prime} y^{\prime}=\Sigma e y^{\prime} z^{\prime}=0
$$

for all axes through the centre of figure; hense for parallel axes through the centre of mass

$$
\Sigma, e x^{\prime} z^{\prime}=\bar{x} \approx \Sigma e,
$$


where $\bar{x}, \vec{y}$ are the coordinates of the centre of figure referred to axes throngh the centre of mass

hence we see

$$
\begin{aligned}
& \bar{x} \Sigma e=\Sigma e x^{\prime} \\
& y \Sigma e=\Sigma e y^{\prime}:
\end{aligned}
$$

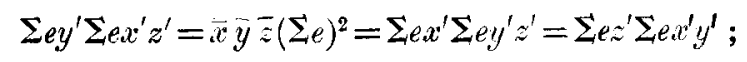

(5) if there is a plane of symmetry in the molecule: for if this plane be taken as the plane $z^{\prime}=0$,

$$
\Sigma e z^{\prime}=0, \Sigma_{e} x^{\prime} z^{\prime}=0, \Sigma e y^{\prime} z^{\prime}=0,
$$

and hence $\mathrm{Q}=0$.

It follows from this that if all the atoms in a molecule are in one plane, the molecule cannot when in solution give rise to optical rotation.

It should be noticed that molecules when in a crystanline arrangement could produce optical rotation when they could not do so in solution, it being assumed that the molecules are not distorted by solution. We have seen that when in solution, either dynamical or electrical symmetry is fatal to rotation. Whereas in the crystalline arrangement rotation would in general exist, unless the molecule was symmetrical dynamically as well as electrically. For rotation to be absent in the crystalline arrangement the coefficients of $l_{1}^{2}, l_{2}^{2}, l_{3}^{2}, l_{1} l_{2}, l_{1} l_{3}, l_{3} l_{3}$ in the expression for $Q($ p. 725$)$ must all vanish. Two important cases in which this condition is fulfilled are (1) when the centre of the electrical charges coincides with the centre of muss ; (2) when the molecule is symmetrical about an axis.

For the present I shall confine myself to the case of rotation in solutions, as this is the one to which the attention of investigators has in the main been directed. As most of these have occupied themselves with substances which contained asymmetric carbon atoms, it is interesting to compare the value and sign of $\bar{Q}$ for two molecules (i.) and (ii.), where (ii.) is such that its masses and electrical charges occupy positions which are the images of their positions in (i.) in a mirror placed in one of the principal planes of (i.). Let us take this plane as the plane $z^{\prime}=0$. The coordinates of the masses and charges on (ii.) will be those of the corresponding mass of (i.) with the sign of the $z^{\prime}$ coordinate

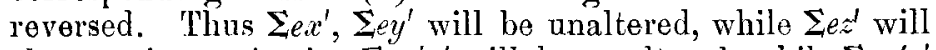
change sign. Again, $\Sigma e x^{\prime} y^{\prime}$ will be unaltered, while $\Sigma e x^{\prime} z^{\prime}$ and $\sum_{e y^{\prime}} z^{\prime}$ will change sign: hence we see that $\bar{Q}$ will have 
the same magnitude in both (i.) and (ii.), but if it is plus for one it will be minus for the other. So that if (i.) rotates the plane of polarization in one, (ii.) will rotate it in the opposite direction. Thus a molecule represented graphically by fig. 1 would rotate in the opposite direction to one represented by fig. 2 , or by fig. 3 .

Fig. 1.

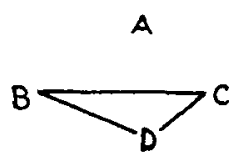

Fig. 2.

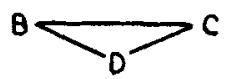

A
Fig. 3.

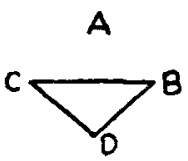

The geometrical meaning of $\bar{Q}$ is interesting. The term

may be written

$$
\Sigma e y^{\prime} \sum_{e} e^{\prime} z^{\prime}-\Sigma_{e} z^{\prime} \sum_{e} x^{\prime} y^{\prime}
$$

$$
\Sigma e_{p} e_{q}\left(x_{p}-x_{q}\right)\left(z_{p} y_{q}-z_{q} y_{p}\right)
$$

where $v_{p}, y_{p}, z_{p} ; x_{q}, y_{q}, z_{q}$ are the coordinates of two points $\mathrm{P}, \mathrm{Q}$ at which the charges are $e_{p}, e_{q}$; the expression may be put in the form

$$
\sum e_{p} e_{q} \mathrm{PQ} \cos \phi . \mathrm{PQ} \sin \phi \cdot p,
$$

where $\phi$ is the angle $\mathrm{PQ}$ makes with the axis of $x$, and $p$ the shortest distance between $P Q$ and this axis. Since $\mathrm{PQ} \sin \phi \cdot p$ is the moment of $\mathrm{PQ}$ about the axis of $x$, and $\mathrm{PQ} \cos \phi$ the component of $\mathrm{PQ}$ along the axis, the term is equal to the product of the charges multiplied by the product of the component of $P Q$ along and its moment about the axis of $x$. Thus it vanishes if $\mathrm{PQ}$ is parallel to the axis, or if it is at right angles to it, or if it intersects it. The term is equal to $e_{p} e_{q} 6 \mathrm{~V}$, where $\mathrm{V}$ is the volume of the tetrahedron whose corners are $P, Q$ and the projections of $P$ and $Q$ on the axis of $x$. The term is to be taken as positive or negative according as the component of $P Q$ along and its moment about the axis of $a$ are related like translation and rotation in a right or left handed screw.

The hypothesis wo have made is that the whole molecule rotates like a rigid body under the influence of the electric forces in a light wave, so that there is no displacement of the electrons relative to the atoms in a molecule. We shall now consider whether on any reasonable supposition as to the values of the quantities involved, the expressions we have found would give values for the rotation of the same order as those observed for optically active substances. 
Consider first the value of $Q$ : the terms $1 / A, 1 / B, 1 / C$, will be of the order $1 / \mathrm{M} d^{2}$, where $\mathrm{M}$ will not be less than the mass of the smallest atom in the molecule, nor greater than the mass of the molecule itself, $d$ is a length comparable with the radius of the molecule. The terms $\Sigma e x^{\prime} y^{\prime}$, $\sum e x^{\prime}$, may for very unsymmetrical molecules be of the order $e d^{2}$ and ed respectively, hence $Q$ will be of the order $e^{2} d / M$ : hence we see from equation (4) that the angular rotation in circular measure per centimetre will be of the order

$$
\frac{\mathrm{N} e^{2} d}{c^{2} \mathrm{M}} \text { or } \mathrm{NM} \cdot \frac{e^{2} d}{c^{2} \mathrm{M}^{2}} \text {. }
$$

When there is one gramme of the substance per cubic cent:metre NM will be less than unity, hence the specific rotation will be less than $e^{2} d / c^{2} \mathrm{M}^{2}$. Now there are many active substances in which the lightest atom is heavier than $\mathrm{OH}$. If we take $M$ to be the mass of this atom, then since $e$ is expressed in electrostatic measure, $e / c \mathrm{M}=10^{4} / 17$, so that the specific rotation per $\mathrm{cm}$. will be less than $d \times 10^{8} / 289$, or taking $d=10^{-8}$ less than $1 / 289, i$. e. less than 12 '. Many opticaliy active substances bave specific rotations greater than $10^{\circ}$, so that our expression for the rotation only accounts for a small fraction of that observed. Nor is this all, the expression we have obtained does not depend upon the frequency of the light, whereas the actual rotations are approximately proportional to the square of the frequency. This discrepancy is due to the fact that we have regarded the molecule as a free system, uninfluenced by other molecules. If the influence of the other molecules is such as to make it set in a definite position and vibrate about this position with a frequency $n$, the displacements and velocities will be less than when the system is free in the proportion of $p^{2}$ to $p^{2}-n^{2}$, where $p$ is the frequency of the light. Thus when the influence of other molecules is to be taken into account, we must multiply the expression we have obtained by $p^{2} / p^{2}-n^{2}$. If $n$ were large compared with $p$, this factor would be $p^{2} / n^{2}$, and as this is proportional to the square of the frequency, we should get the correct variation of the rotation with the frequency of the light. There are, however, two very serious objections to the modification of the formula in this way. In the first place, it is very unlikely that the natural frequency of the motion of a heavy molecule as a whole should be large compared with the frequency of the light; and secondly, even if it were, since the factor $p^{2} / n^{2}$ would then be small, the rotation, as calculated by the 
modified expression, would be much smaller than that calculated by (8) which, as we have seen, is already far too small. Again, from this formula the rotation ought to tend to be smaller for heavier molecules than for light ones; there is no indication of this in the very numerous determinations which have been made of specific rotation.

Thus we do not get sufficient movement to account for the rotation produced by optically active substances if we suppose the whole molecule to rotate as a rigid body under the electric forces in the wave of light. We may, however, regard the molecule as made up of two parts, the first part consisting of the atoms in the molecule, the second of the electrons which bind these atoms together. Thus, with a tetrahedral arrangement of the atoms round the carbon atom, the first system will consist of the carbon atom at the centre $O$ and four other atoms or radicles, A, B, C, D at the corners of the tetrahedron (fig. 4). If we make the

Fig. 4.

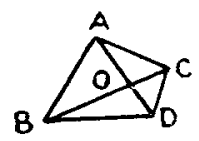

usual assumption that $\mathrm{A}, \mathrm{B}, \mathrm{C}$, and D are bound directly to the carbon atom, then between $O$ and each one of these atoms there must be electrons whose attractions on the positively electrified atoms will bind them together. These structural electrons will form a tetrahedral arrangement which will occupy a definite position relative to the tetrahedron $A, B$, $\mathrm{C}, \mathrm{D}$, and if disturbed from this position will vibrate about it with a definite frequency. When the light waves fall upon the molecule what I imagine to happen is, that in consequence of the relatively enormous mass of the atoms themselves there is but an insignificant displacement of the atomic tetrahedron, but that the much lighter tetrahedral arrangement of the electrons suffers a much greater amount of rotation, and that the motion of the electrons consequent upon this rotation gives rise to the rotation of the plane of polarization.

We have thus to regard the system of electrons as that to which we must apply the preceding analysis. It may be urged against this that since the electrons are all of the same mass, and the electrical centre of the system coincides with the mass centre, the analysis shows that the system would be far.too symmetrical to give rise to optical rotation. This

Phil. Mag. S. 6. Vol. 40. No. 240. Dec. 1920. 3 B 
would be a valid objection if all the electrons were quite free, or even if they were all under similar conditions of restraint. The electrons, however, are not free, they are acted upon by forces which tend to drag them back to the places from which they start. If these forces are different for the different electrons, the behaviour of one electron will be different from that of another, and this may make the system so unsymmetrical that it may be able to produce rotation of the plane of polarization. We may make this clearer by considering a very simple case, that of four electrons $\alpha, \beta, \gamma, \delta$ at the corners of a tetrahedron, and suppose that the restoring forces for two of the electrons, say $\alpha$ and $\beta$, are very large compared with those for $\gamma$ and $\delta$. The effect of this will be much the same as if $\alpha$ and $\beta$ were fixed, so that the tetrahedron would, when acted upon by the electrical forces in a wave of light, rotate about the line $\alpha \beta$, the two electrons $\gamma$ and $\delta$ would rotate about this axis.

We see from the considerations given above that each tetrahedron will contribute to the expression for the rotation of the plane of polarization a term equal to

$$
\frac{2 \pi}{3} \frac{e^{2}}{c^{2}} \frac{[\mathrm{PQ}]}{\mathrm{A}}
$$

where A is the moment of inertia of the two electrons $\gamma$ and $\delta$ about the axis $\alpha \beta$, and [PQ] is six times the volume of the tetrahedron whose corners are $\gamma$ and $\delta$ and the feet of the perpendiculars let fall from $\gamma$ and $\delta$ on $\alpha \beta$. Let us consider the numerical magnitude of this term. If $d$ is a measure of the radius of the molecule, $[\mathrm{PQ}]$ will be of the order $d^{3}$ and A of the order $m d^{2}$, where $m$ is the mass of an electron. Thus the contribution to the rotation will be of the order

$$
\frac{e^{2}}{c^{2}} \frac{d}{m}
$$

and if there are $\mathrm{N}$ molecules per c.c., the rotation will be of the order

$$
\frac{\mathrm{N} e^{2}}{c^{2} m} d
$$

If $M$ is the mass of the molecule, then when there is one gramme of the active substance per c.c., $N M=1$, so that the intrinsic rotation is

Now

$$
\left(\frac{e^{2}}{c^{2} m^{2}}\right)_{\overline{\mathbf{M}}}^{m} d
$$

$$
e^{2} / c^{2} m^{2}=\left(1 \cdot 8 \times 10^{7}\right)^{2}, d=10^{-8} \text {, and } m / \mathrm{M}=\frac{1}{1 \cdot 8 \times 10^{3} \cdot \mathrm{W}},
$$

where $W$ is the molecular weight of the substance. Thus 
the intrinsic rotation would be $\frac{1 \cdot 8 \times 10^{2}}{\mathrm{~W}}$ radians or about $\frac{10^{4}}{W}$ degrees. This has to be multiplied by $p^{2} / n^{2}$, where $n$ is the natural frequency of the vibration of the tetrahedron about the axis $\alpha \beta$. But we see that, even allowing for a small value of $p^{2} / n^{2}$, the system we have considered is able to produce rotations comparable with those excited by optically active substances. If the specific rotation is multiplied by the molecular weight of the active substance, the product is a measure of the rotation due to a single molecule, a quantity which is much more likely to throw light on the properties of the mulecule than the rotation due to a gramme of the substance.

The volume of the tetrahedron which measures the contribution to the optical rotation of one molecule, when the electrons $\gamma \delta$ rotate round $\alpha \beta$, vanishes in the following cases :-

$$
\begin{aligned}
& \text { If } \gamma \delta \text { is parallel to } \alpha \beta \text {. } \\
& \text { If } \gamma \delta \text { intersects } \alpha \beta \text {. } \\
& \text { If } \gamma \delta \text { is at right angles to } \alpha \beta \text {. }
\end{aligned}
$$

From the third of these conditions it follows that no optica! rutation will be produced if $\alpha, \beta, \gamma, \delta$ are at the corners of a regular tetrahedron, there must therefore be some lack of symmetry in the distribution of these electrons. The rotation vanishes whenever $\gamma \delta$ is in a plane at right angles to $\alpha \beta$. Thus it would vanish if the two atoms or radicles which the electrons $\gamma$ and $\delta$ bind respectively to the central carbon atom were identical ; for then by symmetry $a$ and $\beta$ would both be in the plane bisecting $\gamma \delta$ at right angles.

$\AA$ shift in the position of one or both of the electrons $\gamma, \delta$ might change the sign of the optical rotation produced by the molecule. Thus, for example, when the electrons are

Fiv. 5.

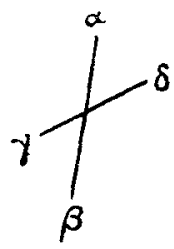

Fig. 6.

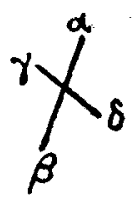

distributed as in fig. $\bar{\jmath}$, the molecule would behave like a positive screw, while if they were as in fig. 6 , it would behave like a negative one. 
We can explain by displacements of this lind the very interesting fact that the optical rotation by a solution of an active substance may depend not merely in magnitude, but also in sign on the nature of the solvent: thus, for examplis, a solution of $d$ acetic acid in water is dextro rotatory, while when dissolved in a mixture of acetone and ether it is laro rotatory (Landolt). If a solvent made some progress towards ionizing the substance by weakening the attachment of one of the atoms (say D) to the carbon atom, it might make the electron $\delta$ which binds $\mathrm{D}$ to the carbon atom nore from $\delta$ to a position more remote from the carbon atom. This change, as fig. 6 shows, might reverse the sign of the rotation.

The general conclusions to which we are led by the proceding investigation, is that the electrical system which is instrumental in producing optical rotation are the electron. which couple the atoms in the molecule to the central carlon atom, and that the most important quantities in the expression: for the rotation are (1) the rigidities of those electrons, i.e. the intensity of the forces restoring them to their position of rest when displaced from it; and (2) the distances of these electrons from the central carbon atom. For it is on these quantities that the dynamical and geometrical asymmetries depend resprectively, and as we have seen, both these asymmetries are essential for rotation in solutions. If it were not for differences in (1) there would be no dynanic asymmetry, for the electrons have all the same mas. When, however, they are pulled back when displaced from their position of equilibrium with forces of different intensities, the effect will be much the same as if the elcctrons had different masses. Thus, if the frequency of the vibrations of the electron when displaced from its position of equilibrium is $n_{1}$ and $p$ is the frequency of the light, the behaviom of the electron under the forces in the light, wave will be much the same as if the electron were free but had a mass $\left(p^{2}-n_{1}^{2}\right) / p^{2}$ times itsinormal mass. When $n_{1}^{2}$ is large compared with $p$, the effective mass is approximately $n_{1}^{2} / p^{2}$ times the normal mass, and is thus much greater. Thus the dynamical asymmetry of the molecule will be measured, not as on Gnye's theory * by differences in the atomic weights of the atoms attached to the carbon atom, for these on our view have no direct bearing on the rotation, but on the differences in the frequencies of the electrons which bind the atoms to the carbon atom.

$$
\text { * Ann. Chim. Phys. (6) xxv. p. } 145 \text { (1892). }
$$


A wain, the geometrical asymmetry which is necessary for roiation will depend on the distances of these electrons from the carbon atom. Thus, to predict the rotation produced by a molecule containing one unsymmetrical carbon atom and represented by $C_{1} R_{2} R_{3} R_{4}$, we require to know the properties of the linkages $\mathrm{CR}_{1}, \mathrm{CR}_{2}, \mathrm{CR}_{3}, \mathrm{CR}_{4}$; i. e., we require to know the frequency of the coupling electron in the linkage CR and its distance from C:

Unfortunately our knowledge on these points is extremely meagre. Wre might expect to get some knowledge of the frequencies by mapping the absorption spectra of various hydrocarbons. Thus, if the coupling ( $-\mathrm{H}$ had a frequency $n_{1}$, we might expect to find in the absorption spectrum of a compound containing this coupling a band corresponding to this firequency, modified it night be by the proximity of other couplings in the molecule. A study of the spectra of compounds containing the coupling ( $-\mathrm{OH}$ might, in a similar way, lead to the knowledge of the frequency of the electrons concerned in this coupling. And in this way, by the study of the absorption spectra of a large number of orowic compounds, we might hope to determine the frequency of the coupling of the carbon atom with the various atoms and radicles to which it is joined in organic compounds. Considerable progress has been made in the study of such absorption spectri. Thus Hartley (see Kayser's 'Spectroscopy,' vol. iii.), working with ultra-violet light down to the wave-length 2000 , studied the absorption in the ultraviolet down to this limit, and discovered well-defined absorption bands in benzene and other aromatic compounds containing the benzene ring. Onr knowledge of this subject has been greatly extended by Stark and his collaborators who, by using fluorite lenses and prisms, were able to work with ultra-violet light down to wave-length 1850 , and discovered, among other things, bands which they ascribed to the linkages $\mathrm{C}=\mathrm{C}$ and $\mathrm{C} \equiv \mathrm{C}$. We are however not yet, I think, in a position to be able to say what are the fundamental frequencies of the various linkages; to do so we require observations over a wider range of frequencies extending to wave-lengths considerably smaller than those hitherto studied, for in this, the Schum?nn region, nearly all gases have great absorption, and some of it may well be selective. The experiments hitherto made indicate that the frequencies corresponding to the linkages $\mathrm{C}-\mathrm{H}$ and $\mathrm{C}-\mathrm{OH}$ will be very far in the ultra-violet, and consequently larger than the normal value. Now, on the view we have taken, a high frequency corresponds to a large effective mass, so that in 
compounds of the type C.H.HO, $\mathrm{R}_{1}, \mathrm{R}_{2}$, the system of coupling electrons will tend to twist round the line joining the electrons which couple $\mathrm{H}$ and $\mathrm{OH}$ with the carbon atom.

The other condition for optical rotation is geometrical asymmetry. The electrons cannut be at the corners of a regular tetrahedron, and this arrangement, which is the one usually asssumed, is incompatible-with optical rotation. The departure of the molecule from this form, in fact the shape of the molecule, is of vital importance in connexion with optical rotation, but on this subject little, if anything, seems to have been done.

If we had a theory which gave the configuration of the molecule and the periods of vibrations of the electrons, we could calculate by the expression given above the value of the molecular rotation. Inasmuch as the configuration and periods enter into these expressions in a complicated way, the effect of any one period, for example, depending on its relation to each of the other periods, the periods are not easily calculated from the rotation. Thus observations on the optical rotation are more likely to be useful as a test of any theory of the configuration and structure of the molecule than as a means of discovering this structure.

Since the above was written I have seen a paper by Stark (Jahr. f. Radioaktivität, xi. p. 194, 1914), in which the subject of optical rotation is also treated from the point of view of the electron theory; the treatment is wholly qualitative, and I find it difficult to follow the reasoning; so far as $\mathrm{I}$ am able to do so it seems to me to be fundamentally different from that given above.

LXXXIV. The Charge on the Atomic Nucleus and the Law of Force. By J. Chadwick, M.Sc., Wollaston Student of Gonville and Caius College, Cambridge*.

$\S 1$. $7 \mathrm{HE}$ theory of the nuclear constitution of the atom, 1 put forward by Sir Ernest Rutherford $†$ in 1911, has been confirmed by evidence gathered from such various sources that it now forms the foundation on which the development of atomic physics is based. On this theory, the positive charge associated with an atom is concentrated on a massive nucleus of small dimensions, surrounded

* Commanicated by Professor Sir E. Rutherford, F.L.S.

+ Rutherford, Phil. Mag. xxi. p. 669 (1911). 